\title{
Determinación de parámetros críticos y análisis exploratorio de datos del proceso de añejamiento de ron
}

\section{Critical parameters determination and data exploratory analysis of rum aging process}

\section{Beatriz García-Castellanos ${ }^{1}$, Osney Pérez-Ones², Lourdes Zumalacárregui de Cárdenas ${ }^{3}$, Idania Blanco-Carvajal ${ }^{4}$, Luis Eduardo López de la Maza ${ }^{5}$}

Fecha de recepción: 19 de setiembre de 2019

Fecha de aprobación: 22 de enero de 2020

García-Castellanos, B; Pérez-Ones, O; Zumalacárregui de Cárdenas, L; Blanco-Carvajal, I; López de la Maza, L. Determinación de parámetros críticos y análisis exploratorio de datos del proceso de añejamiento de ron. Tecnología en Marcha. Vol. 33-4. Octubre-Diciembre 2020. Pág 26-36.

doi https://doi.org/10.18845/tm.v33i4.4692

1 Ingeniera Química, Centro de Referencia de Alcoholes y Bebidas (CERALBE), Instituto Cubano de Investigaciones de los Derivados de la Caña de Azúcar (ICIDCA). La Habana, Cuba. Correo electrónico: beatriz.garcia@icidca.azcuba.cu. (iD https://orcid.org/0000-0001-8101-0638

2 Profesor Auxiliar, Decano, Facultad de Ingeniería Química, Universidad Tecnológica de La Habana "José Antonio Echeverría". La Habana, Cuba. Correo electrónico: osney@quimica.cujae.edu.cu. (D) https://orcid.org/0000-0002-0366-0317

3 Profesor Titular, Facultad de Ingeniería Química, Universidad Tecnológica de La Habana "José Antonio Echeverría". La Habana, Cuba. Correo electrónico: Iourdes@quimica. cujae.edu.cu.

4 Jefe de Producción, Centro de Referencia de Alcoholes y Bebidas (CERALBE), Instituto Cubano de Investigaciones de los Derivados de la Caña de Azúcar (ICIDCA). La Habana, Cuba. Correo electrónico: idania.blanco@icidca.azcuba.cu. (iD https://orcid.org/0000-0003-1281-3722

5 Profesor Instructor, Facultad de Ingeniería Química, Universidad Tecnológica de La Habana "José Antonio Echeverría". La Habana, Cuba. Correo electrónico: llopezm@quimica.cujae.edu.cu. 


\title{
Palabras clave
}

Añejamiento de rones; validación de procesos; parámetros críticos; atributos críticos; análisis de componentes principales.

\section{Resumen}

El proceso de añejamiento de ron experimenta pérdidas de volumen, denominadas mermas. Como parte de la validación continuada del proceso se empleó el programa Statgraphics Centurion v17.2 para el Análisis de Pareto determinándose los parámetros críticos: tiempo de añejamiento, volumen, concentración de dioxígeno; temperatura y humedad. El buen funcionamiento del proceso está influenciado entre otros factores, por las variables de operación, las cuales se registran en bases de datos. El análisis de componentes principales (ACP) a partir de dichas bases de datos, permitió obtener conocimiento del comportamiento de las variables más influyentes en el añejamiento usando la herramienta The Unscrambler v10.4. Con el ACP quedaron definidas las variables que se miden más importantes en el proceso: temperatura, humedad, tiempo de añejamiento, grado alcohólico y volumen. Se redujo la dimensionalidad a tres componentes principales que explican el 89,11\% de la varianza total de los datos e incluyen las cinco variables. Se calcularon las mermas en bodega durante los 13 meses de estudio, que resultaron ser 763,43 L, lo cual equivale a un 10,5 \% del volumen inicial existente; superando el 6 \% permitido en la norma de la República Dominicana, considerada como referencia en este trabajo. Esta merma representa una pérdida de $\$ 32718$. Se determinó que el $6 \%$ de mermas con respecto al volumen inicial de la solera es 434,72 L, equivalentes a pérdidas de \$18 631 y a una disminución de \$14 087 con respecto a las existentes actualmente.

\section{Keywords}

Rum aging; process validation; critical parameters; critical attributes; principal component analysis.

\begin{abstract}
Volume losses, known as wastage are presented during the rum aging process. As part of the continuous validation of the process Pareto Analysis is applied to determine the critical parameters that affect it. They are: aging time, volume, dioxygen concentration; temperature and humidity. The proper functioning of the process is influenced, among other factors, by the operation variables, which are recorded in databases. In this work, the databases were used to carry out a principal component analysis (PCA). The behavior of the main variables that affect the aging was understood, applying PCA, using the software The Unscrambler v10.4. With the $\mathrm{ACP}$, the more important variables of the process were defined. The dimensionality was reduced to three main components that explain $89,11 \%$ of the total variance of the data, including the five variables. Wastages during 13 months were calculated (763.43 L). This value represents $10.5 \%$ of the initial volume, higher than the $6 \%$ recommended by the Dominican Republic Regulation, considered as a reference in this research. This wastage represents losses equivalent to \$32 718. Losses that correspond to the $6 \%$ of the initial volume were calculated ( $\$ 18631)$. If the process fulfils the Dominican regulation, $\$ 14087$ could be saved.
\end{abstract}




\section{Introducción}

En el ron fresco, como en la mayoría de las bebidas alcohólicas destiladas, el aroma recuerda a la materia prima utilizada. Este varía cuando se deja en reposo en recipientes de roble por un tiempo determinado, es decir durante el proceso de permanencia de los aguardientes en barriles de roble y al cual se le conoce comúnmente como "envejecimiento" o tiempo de añejamiento. Este tiempo permite que las bebidas sufran una evolución que repercute decisivamente en la mejoría de sus características y propiedades organolépticas [1].

Durante el proceso de añejamiento del ron se producen pérdidas de producto. El añejamiento del ron no cambia ni transforma la bebida, sino que desarrolla y sublima sus cualidades latentes. Es por ello que, en el contexto de excelencia en que compiten estas bebidas, surge el interés por estudiar la merma en el volumen de ron durante su añejamiento en relación a las condiciones ambientales existentes en la solera.

En el mundo el valor medio de pérdidas anuales que se considera para toneles de 180 litros es de $10 \%$ del volumen almacenado. Sin embargo, la literatura señala para los países del Caribe $13 \%$ de pérdidas anuales [2]. En un trabajo realizado en las soleras de la ronera de Cárdenas, en los años 1985-1986, se corroboró una pérdida de 10,5 \% anual. No se han encontrado reportes recientes del valor de estas pérdidas en Cuba. En Jamaica se reporta un 6\% de pérdidas [3].

La tecnología existente en las bodegas permitió llevar a cabo un estudio del comportamiento de las mermas durante 13 meses de añejamiento. Se realizaron mediciones del nivel del líquido de los toneles, grado alcohólico, temperatura y humedad. Toda esta memoria almacenada constituye una valiosa fuente de información que puede ser útil en la comprensión del presente y en la predicción del futuro [4]. Se tienen así, cálculos desactualizados y altos porcentajes de mermas en volumen de las bases en solera con desconocimiento de los factores que influyen.

\section{Materiales y métodos}

\section{Verificación continuada de procesos}

Desde hace varias décadas la validación ha sido uno de los aspectos de mayor escrutinio dentro de las buenas prácticas de fabricación como vía principal para el aseguramiento de los procesos. En el enfoque actual la validación del proceso consiste en: "recolectar y evaluar información del proceso desde su etapa de diseño hasta la producción, que establece evidencia científica de que el mismo es capaz de entregar un producto de calidad de forma consistente" [5].

El ciclo de vida de validación cuenta con tres etapas: diseño del proceso, calificación del proceso y verificación continuada [5]. La verificación continuada del proceso es la tercera y última etapa dentro del ciclo de vida de validación. Es una alternativa al enfoque tradicional de validación de procesos, donde una vez concluida la calificación del proceso productivo, se monitorea y evalúa sistemáticamente su mantenimiento del estado de control. En el desarrollo de un nuevo producto o de uno ya existente es necesario dominar el proceso conociendo cómo los materiales, procesos y controles afectan el producto final, ya que se puede identificar los factores críticos de entrada al proceso. La variación en las respuestas del proceso debe ser entendida y controlada. Desde una perspectiva de fabricación, las materias primas y los parámetros del proceso afectan en mayor o menor medida los atributos críticos de calidad.

Para conocer cuáles parámetros del proceso son críticos y cuáles claves, conocer en qué dirección y magnitud estos parámetros afectan los atributos críticos de calidad es fundamental para la excelencia de fabricación. Esta comprensión puede ser utilizada para desarrollar procesos más robustos o añadir medidas de control adecuadas para ajustar los procesos y así obtener el producto objetivo [6]. 
Procedimiento de cálculo de mermas en toneles de añejamiento de ron

Para la realización de este estudio se instaló un dispositivo de medición de temperatura y humedad relativa ambiente marca HOBO, modelo H08-003-02 con registrador de datos en una solera. Estas variables físicas se midieron ininterrumpidamente durante catorce meses.

Los toneles se hallan almacenados en cinco ramblas que poseen una distribución vertical de cinco niveles cada una; en ellas se observa la identificación numérica de cada tonel.

Se escogieron tres puntos de la solera (centro y extremos) y se midieron los toneles del primer, tercer y quinto nivel, para evaluar las pérdidas en relación a la posición y la altura de los mismos. Se escogieron 45 toneles los cuales tienen almacenados diferentes bases.

El dispositivo de medición se instaló sobre las duelas de uno de los barriles del nivel más elevado de la rambla, en las cercanías del tapón utilizado para el llenado/vaciado de la barrica y del techo de la solera.

Los toneles contienen cinco (cinco) productos con diferentes concentraciones de etanol (formulaciones) y se encuentran distribuidos aleatoriamente en la solera; denominados: B Vi, B Va, B A, Ag, V V [7].

\section{Análisis de Pareto}

El diagrama de Pareto es una herramienta estadística que permite organizar por orden de relevancia los problemas o las causas que los generan. La utilización de este diagrama permite que cuando se quiera mejorar un proceso o atender sus problemas se establezcan prioridades y se enfoquen los esfuerzos donde puedan tener mayor impacto.

El viejo adagio dice que el $80 \%$ de los problemas reportados se asocia con el $20 \%$ de las diversas causas esenciales. Concentrando los esfuerzos en corregir este $20 \%$, se puede tener el mayor impacto inmediato en la calidad del producto. En esta investigación fue elaborado utilizando la herramienta Statgraphics Centurion v17.2.

\section{Análisis de componentes principales (ACP)}

El ACP es una técnica que facilita el análisis exploratorio de datos al explicar las fuentes de variabilidad, reducir dimensionalidad, eliminar datos discrepantes y extraer la información importante. En el trabajo se utilizó el software The Unscrambler X v10.4, que es una herramienta integral de análisis de datos para estadística exploratoria, análisis multivariado, clasificación, predicción y diseño de experimentos [4].

\section{Resultados}

Determinación de los atributos críticos de calidad

Se clasificaron los atributos críticos de calidad de acuerdo al impacto sobre la calidad del producto final y el riesgo potencial sobre la aceptación del lote. Para ello se empleó el cuadro 1 de asignación de índices, obteniéndose los resultados que se muestran en el cuadro 2 [6], [8].

Para la determinación de los parámetros críticos se formó una comisión de seis expertos, integrada por másteres y profesionales en las especialidades de ciencia y tecnología de los procesos biotecnológicos, ingeniería de alimentos y química analítica; y se analizó un conjunto de variables que, según la experiencia de trabajo y el conocimiento del proceso, tenían mayor o menor influencia en los atributos de salida del producto final. Se tuvo en cuenta en este análisis solo la etapa de añejamiento. 
Cuadro 1. Asignación de índices para los atributos críticos de calidad

\begin{tabular}{|l|c|c|}
\hline \multicolumn{1}{|c|}{ Elementos de valoración } & $\begin{array}{c}\text { Nivel de } \\
\text { criticidad }\end{array}$ & Índice \\
\hline $\begin{array}{l}\text { El atributo tiene impacto potencial sobre el desempeño de los procesos posteriores e } \\
\text { incluso puede reflejarse negativamente en la calidad del producto final sin capacidad de } \\
\text { ser detectado. }\end{array}$ & Muy alto & 9 \\
\hline $\begin{array}{l}\text { El atributo tiene impacto potencial sobre el desempeño de los procesos posteriores e } \\
\text { incluso puede reflejarse negativamente en la calidad del producto final, aunque con } \\
\text { capacidad de ser detectado. }\end{array}$ & Alto & 7 \\
\hline $\begin{array}{l}\text { El atributo tiene impacto potencial sobre el desempeño de los procesos posteriores, aun- } \\
\text { que sin repercusión negativa en la calidad del producto final. }\end{array}$ & Moderado & 5 \\
\hline El atributo no tiene impacto significativo sobre el desempeño de los procesos posteriores. & Bajo & 3 \\
\hline El atributo no tiene impacto sobre el desempeño de los procesos posteriores. & Muy bajo & 1 \\
\hline
\end{tabular}

Cuadro 2. Resultados de asignación de índice para cada atributo crítico de calidad del ron

\begin{tabular}{|c|c|c|c|c|}
\hline $\begin{array}{l}\text { Atributos críticos } \\
\text { de calidad }\end{array}$ & Método & $\begin{array}{l}\text { Criterio de } \\
\text { aceptación }\end{array}$ & $\begin{array}{l}\text { Nivel de } \\
\text { criticidad }\end{array}$ & Argumentación y observaciones \\
\hline $\begin{array}{l}\text { Grado alcohólico } \\
\left(\% \text { v/v a } 20^{\circ} \mathrm{C}\right)\end{array}$ & $\begin{array}{l}\text { Densimetría } \\
\text { digital }\end{array}$ & $\begin{array}{l}\text { 39,5-40,5 (Añejo) } \\
\text { 39,4-40,6 } \\
\text { (Reserva) }\end{array}$ & 7 & $\begin{array}{l}\text { Se determina y ajusta para la etapa de } \\
\text { formulación }\end{array}$ \\
\hline $\begin{array}{c}\text { Acidez total ( } \mathrm{g} \text { de } \\
\text { ácido acético/100 } \\
\mathrm{L} \text { de etanol } \\
\left.100^{\circ} \mathrm{GL}\right)\end{array}$ & $\begin{array}{l}\text { Valoración con } \\
\text { sosa }\end{array}$ & $\begin{array}{c}\text { 70-130 (Añejo) } \\
\text { 80-150 (Reserva) }\end{array}$ & 5 & $\begin{array}{l}\text { Se determina y ajusta para la etapa de } \\
\text { formulación }\end{array}$ \\
\hline $\begin{array}{c}\text { Componentes } \\
\text { mayoritarios } \\
\text { (g/100 L de etanol } \\
\left.100^{\circ} \mathrm{GL}\right)\end{array}$ & $\begin{array}{l}\text { Cromatografía de } \\
\text { gases }\end{array}$ & $\begin{array}{l}\text { 30-60 (Acetato } \\
\text { de etilo) 70-100 } \\
\text { (Alcoholes } \\
\text { superiores) 5-15 } \\
\text { (Acetaldehído) }\end{array}$ & 3 & $\begin{array}{l}\text { Se debe tener definido y cumplir con el } \\
\text { intervalo establecido }\end{array}$ \\
\hline $\begin{array}{l}\text { Color(expresado } \\
\text { en unidades de } \\
\text { densidad óptica) }\end{array}$ & Espectrofotometría & $\begin{array}{l}\text { 0,7-1,1 (Añejo y } \\
\text { Reserva) }\end{array}$ & 3 & $\begin{array}{l}\text { Debe presentar color ámbar. Ser brillante, } \\
\text { limpio y transparente. Se ajusta con el color } \\
\text { caramelo }\end{array}$ \\
\hline $\begin{array}{l}\text { Composición } \\
\text { organoléptica }\end{array}$ & $\begin{array}{l}\text { Técnica IIIA } \\
\text { (Instituto de } \\
\text { Investigaciones } \\
\text { de la Industria } \\
\text { Alimentaria) }\end{array}$ & $\begin{array}{l}\text { Debe cumplir } \\
\text { con las } \\
\text { características } \\
\text { predefinidas del } \\
\text { ron añejo }\end{array}$ & 9 & $\begin{array}{l}\text { Aroma propio de un ron añejo ligero, típico } \\
\text { de los rones oscuros bien añejados, seco. } \\
\text { Integración clara de los rones oscuros } \\
\text { bien añejados que participan. Correcta } \\
\text { evolución de los aromas provenientes de la } \\
\text { destilación con marcada preponderancia } \\
\text { de los del añejamiento. }\end{array}$ \\
\hline $\begin{array}{l}\text { Presencia de } \\
\text { metales }\end{array}$ & $\begin{array}{l}\text { Fotómetro de } \\
\text { Ilamas }\end{array}$ & Ausencia & 9 & $\begin{array}{l}\text { Se realiza para determinar la presencia de } \\
\text { metales que puedan precipitar. }\end{array}$ \\
\hline
\end{tabular}


En la valoración numérica para cada parámetro de proceso se empleó el cuadro 3 de asignación de índices y con los valores de criticidad asignados a cada atributo crítico de calidad se determinó la suma de los productos por cada parámetro en la fila de la matriz correspondiente y su porcentaje respecto al gran total.

Cuadro 3. Asignación de índices para los parámetros críticos de calidad

\begin{tabular}{|l|c|c|}
\hline \multicolumn{1}{|c|}{ Elementos de valoración } & $\begin{array}{c}\text { Nivel de } \\
\text { impacto }\end{array}$ & Índice \\
\hline $\begin{array}{l}\text { La variabilidad incide directamente sobre el atributo crítico de calidad, incluso sobrepasando } \\
\text { etapas intermedias del proceso donde no ocurren transformaciones asociadas al parámetro } \\
\text { y/o variable en cuestión. Existen evidencias y/o experiencia de que existe una alta correlación. }\end{array}$ & Muy alto & 9 \\
\hline $\begin{array}{l}\text { La variabilidad incide indirectamente sobre el atributo crítico de calidad, dado que puede afec- } \\
\text { tar etapas intermedias del proceso cuyo desempeño influye de forma apreciable sobre dicho } \\
\text { atributo. Existen evidencias y/o experiencia de que existe una correlación aún significativa. }\end{array}$ & Alto & 7 \\
\hline $\begin{array}{l}\text { La variabilidad incide en el desempeño de etapas del proceso, aunque con menor inciden- } \\
\text { cia sobre el atributo crítico de calidad. Existen evidencias y/o experiencia de que la correl- } \\
\text { ación moderada. }\end{array}$ & Moderado & 5 \\
\hline $\begin{array}{l}\text { La variabilidad incide en el desempeño de etapas del proceso, aunque con poca o ninguna } \\
\text { incidencia sobre el atributo crítico de calidad. Existen evidencias y/o experiencia de que la } \\
\text { correlación es baja. }\end{array}$ & Bajo & 3 \\
\hline $\begin{array}{l}\text { La variabilidad incide poco en el desempeño de etapas de proceso sin afectar ningún atribu- } \\
\text { to crítico de calidad. Existen evidencias y/o experiencia de que no existe correlación alguna. }\end{array}$ & Muy bajo & 1 \\
\hline
\end{tabular}

En el cuadro 4 se muestra la matriz de valoración de los resultados obtenidos.

Cuadro 4. Matriz de valoración

\begin{tabular}{|c|c|c|c|c|c|c|c|c|c|}
\hline \multicolumn{2}{|c|}{$\begin{array}{c}\text { Parámetros de operación / } \\
\text { Atributos de calidad }\end{array}$} & \multirow{2}{*}{$\begin{array}{c}\frac{0}{0} \\
\frac{0}{0} \\
\frac{\pi}{0} \frac{0}{0} \\
\frac{0}{\sigma} \\
\frac{0}{\sigma}\end{array}$} & \multirow{2}{*}{$\begin{array}{l}\bar{\sigma} \\
\frac{\pi}{0} \\
N \\
\frac{0}{0} \\
\frac{0}{0} \\
\frac{0}{4} \\
5\end{array}$} & \multirow{2}{*}{ 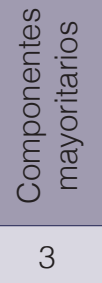 } & \multirow{2}{*}{$\begin{array}{l}\frac{\overline{0}}{0} \\
0 \\
3\end{array}$} & \multirow{2}{*}{ 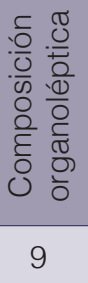 } & \multirow{2}{*}{ 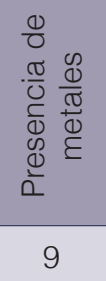 } & \multirow{2}{*}{ 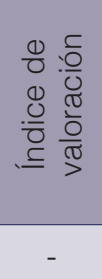 } & \multirow{2}{*}{$\begin{array}{l}\overline{\widetilde{\pi}} \\
\stackrel{0}{0} \\
0 \\
\text { o }\end{array}$} \\
\hline Id & Nivel de criticidad & & & & & & & & \\
\hline 1 & Humedad & 9 & 7 & 7 & 1 & 7 & 3 & 300 & 12,51 \\
\hline 2 & Temperatura & 9 & 7 & 7 & 1 & 7 & 3 & 228 & 12,51 \\
\hline 3 & Tiempo de añejamiento & 5 & 9 & 9 & 7 & 7 & 9 & 222 & 17,71 \\
\hline 4 & Grado alcohólico inicial & 9 & 9 & 9 & 3 & 3 & 3 & 212 & 10,04 \\
\hline 5 & Volumen & 9 & 9 & 9 & 7 & 5 & 3 & 212 & 13,46 \\
\hline 6 & Concentración de $\mathrm{O}_{2}$ & 9 & 9 & 9 & 5 & 5 & 3 & 188 & 13,11 \\
\hline 7 & Quemado del barril (confección) & 3 & 7 & 7 & 7 & 7 & 3 & 170 & 11,10 \\
\hline 8 & Hinchado del tonel (con agua) & 3 & 3 & 3 & 3 & 3 & 9 & 162 & 9,56 \\
\hline \multicolumn{8}{|c|}{ Gran total } & 1694 & 100 \\
\hline
\end{tabular}


En la figura 1 se muestra el Diagrama de Pareto, representando además la línea ascendente del porcentaje acumulativo para cada parámetro.

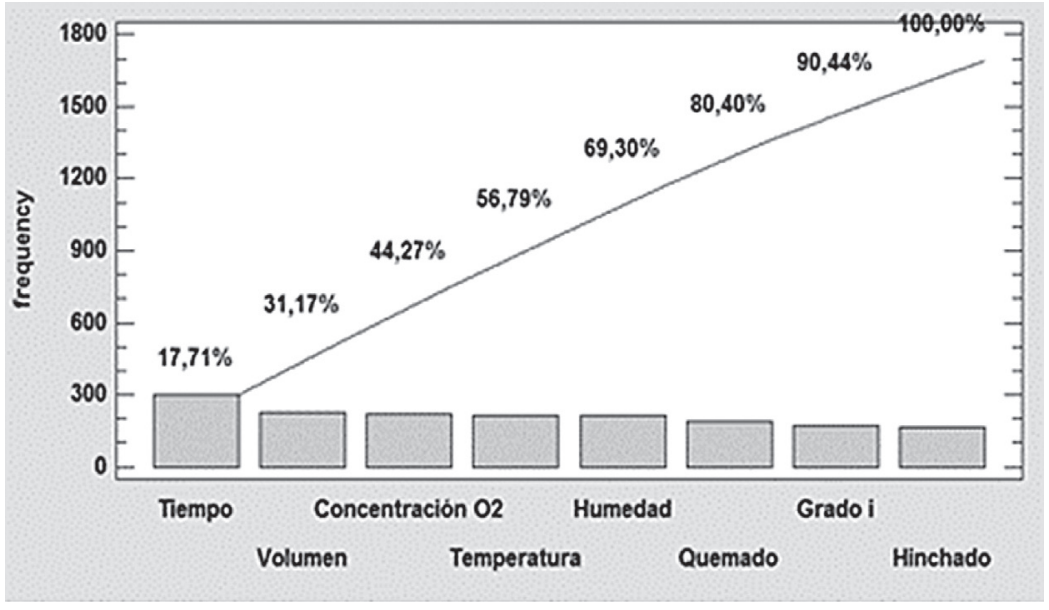

Figura 1. Diagrama de Pareto

En la figura 1 se observan las variables que tienen mayor influencia en los atributos críticos de calidad del producto final. Esto se justifica a partir del criterio de Pareto $80-20 \%$, donde estas variables representan los parámetros que necesitan de un control estricto para así garantizar que los atributos críticos de calidad del ron, se mantengan en las especificaciones establecidas (los que las tengan). Estos son los llamados parámetros críticos del proceso (CPP), y son: tiempo de añejamiento, volumen, concentración de dioxígeno; temperatura y humedad. Las variables: grado alcohólico inicial, quemado e hinchado del tonel son los parámetros claves del proceso (KPP), ya que influirán de cierta forma en el desempeño del proceso, pero no en los atributos críticos de calidad.

En este estudio queda recogida la mayor parte de la información teórica y las experiencias productivas de los especialistas y operarios del área. Para el estudio posterior se decidió excluir la variable concentración de dioxígeno debido a que no se cuenta con la tecnología apropiada para medirla. Además, se agregó al análisis la variable grado alcohólico inicial ya que su valoración se realizó teniendo en consideración que puede ser ajustado para las especificaciones finales del proceso y no por el impacto específico a lo largo de este.

Determinación de ruido, limpieza y selección de los datos que se utilizaron

Se contó con un conjunto de datos integrado por 900 instancias y diez variables, de ellas cinco cualitativas: producto, rambla, fecha y posiciones horizontal y vertical; mientras que las restantes: tiempo de añejamiento, volumen, temperatura, humedad y grado alcohólico inicial tienen carácter cuantitativo. La matriz inicial de datos se redujo a 546 valores ya que presentaba mediciones incompletas con respecto a tres toneles $(19,21$ y 159) y al período de tiempo que comprende de febrero a septiembre de 2014.

\section{Análisis estadístico y transformación de los datos}

El hecho de que todas las variables se hayan registrado por su propia naturaleza justifica que se hayan escalado por el rango y centrado por la media. El ACP se realizó partiendo de un fichero de datos con las variables producto, rambla, posiciones horizontal y vertical, fecha, volumen, grado alcohólico, temperatura, humedad y tiempo de añejamiento. 
El gráfico de la influencia para este ACP se puede observar en la figura 2.

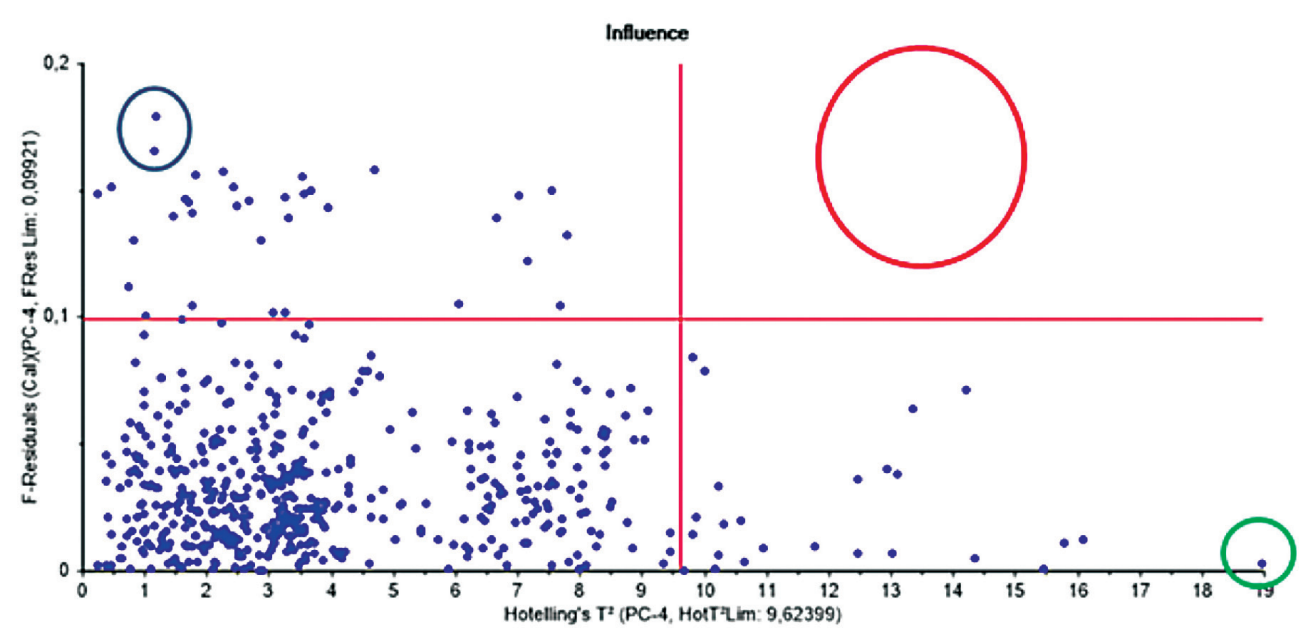

Figura 2. Gráfico de la influencia resultado del ACP al fichero con 10 variables y 546 instancias

Se puede observar que no existen muestras que constituyan puntos discrepantes peligrosos, los que de existir aparecerían en el espacio representado por el círculo rojo ubicado en la sección superior derecha del gráfico.

En el gráfico anterior se puede observar que las muestras 209 y 210 (enmarcadas por el círculo azul) tienen alto valor residual por lo que constituyen puntos discrepantes pero no son peligrosos y pudieran contener información valiosa. Además, este punto se encuentra dentro de la elipse de Hotelling mostrada más abajo en la figura 3; por tanto, esta muestra no fue eliminada del análisis.

La muestra 390 (enmarcada por el círculo verde) presenta un elevado valor de distanciamiento por lo que constituyen un punto discrepante influyente, pero contiene información valiosa y por tanto no fue eliminada del análisis.

Para complementar el análisis de los puntos discrepantes y descubrir agrupamientos no perceptibles fácilmente se utilizó el gráfico de las puntuaciones con la elipse de Hotelling (figura 3). En este se puede observar cómo la mayoría de los puntos se encuentran dentro de la elipse, evidenciando que todos pertenecen al mismo proceso. Además, se muestra un agrupamiento fuera de la elipse; en la parte inferior. Estas puntuaciones discrepantes corresponden a muestras del producto Ag que presentan la mayor graduación alcohólica de la base de datos, que se encuentran ubicadas en la rambla tres y en la posición horizontal inferior (piso). Esto está directamente relacionado con los cambios abruptos experimentados, en estas instancias de tiempo, por la humedad ambiental, porque en la medida que esta sea mayor o menor se va a favorecer la evaporación de etanol o de agua.

Para analizar el comportamiento de la variable mermas de acuerdo al gráfico de las puntuaciones, se determinaron los valores de la media y la desviación estándar y se estableció que los valores de esta variable menores que el límite inferior eran ideales, los menores que la media eran normales y los mayores que el límite superior eran altos. De esta manera se pudo identificar la zona de mayores valores de mermas, ubicada a la izquierda del gráfico y en color verde, perteneciente al período más caluroso del año en especial a septiembre de 2015; alcanzándose valores extremos de temperatura para el proceso en el intervalo de (34-37) ${ }^{\circ} \mathrm{C}$. 


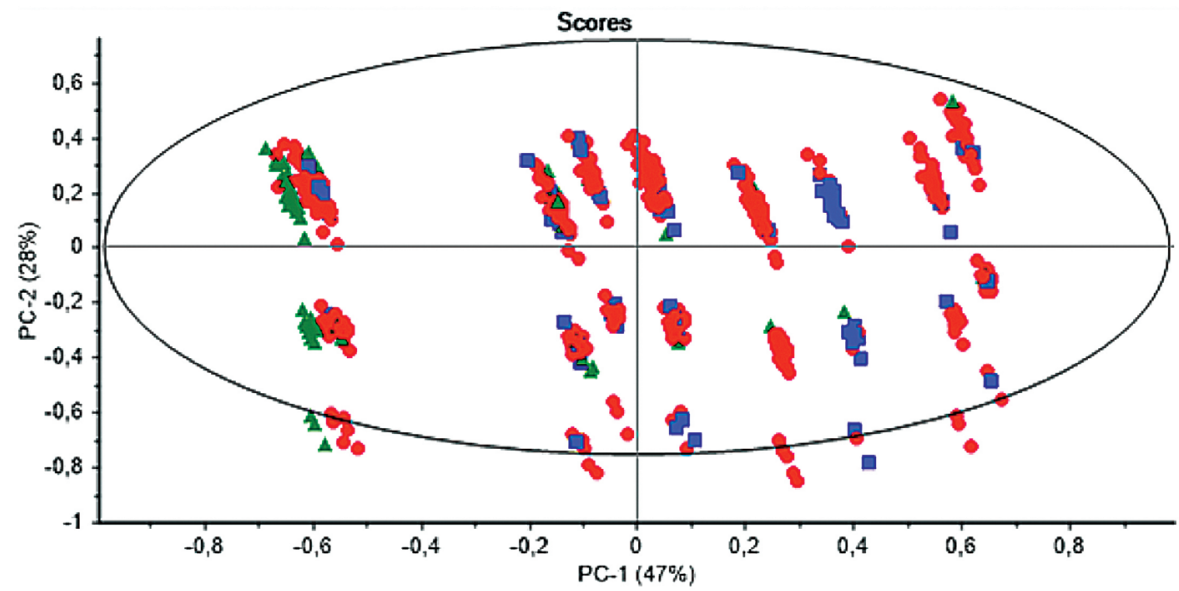

Figura 3. Gráfico de las puntuaciones con elipse de Hotelling resultado del ACP al fichero con 10 variables y 546 instancias

Tres componentes principales logran explicar el 88,32\% de la varianza de los datos iniciales. En la figura 4 se observa el gráfico de la varianza explicada. El primer componente explica el 46,43 $\%$ de la varianza el segundo el 27,34 \% y el tercero el 14,55 \%. Si se toma en consideración un mayor número de componentes se lograría explicar el 95,90 \% de la varianza, pero el modelo sería más complejo.

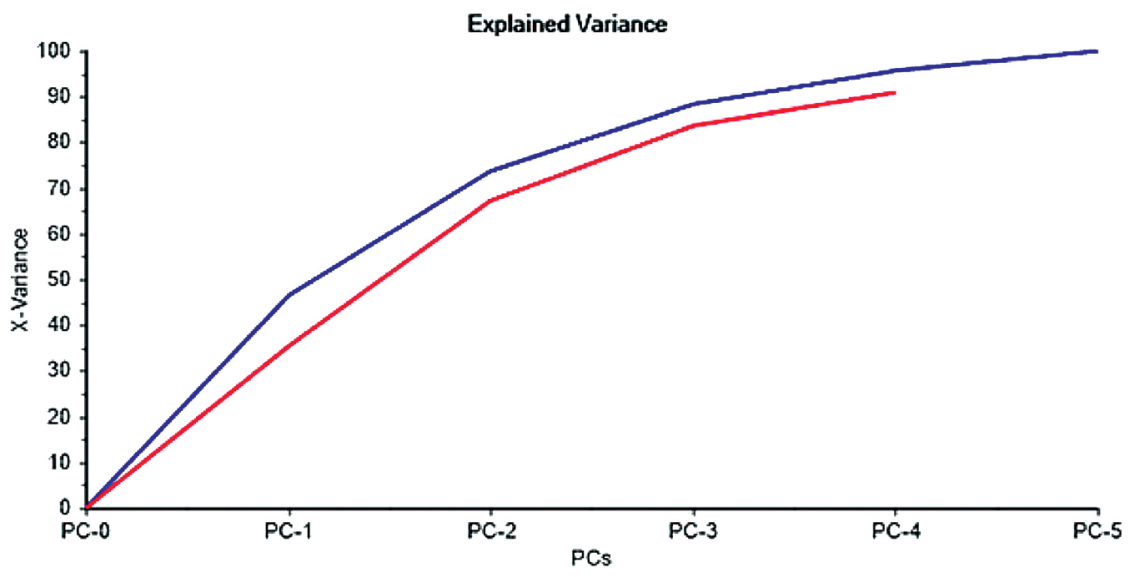

Figura 4. Gráfico de la varianza explicada resultado del ACP al fichero con 10 variables y 546 instancias

El gráfico de los pesos correlacionados muestra una mejor visualización del comportamiento de las variables. En la figura 5 se presenta el gráfico de los pesos correlacionados.

Como se puede observar en la figura 5 con excepción del volumen, todas las variables se encuentran entre las dos elipses, por lo que todas son significativas ya que tienen un elevado aporte al primer y segundo componente que logran explicar el 74,64 \% de la varianza total inicial. Este resultado justifica la inclusión de todas estas variables para la modelación posterior. La variable volumen al presentar el menor coeficiente variación (7,63 \%) justifica que no se incluya entre las dos elipses que explican entre el 50 y $100 \%$ de la varianza total de los datos. 


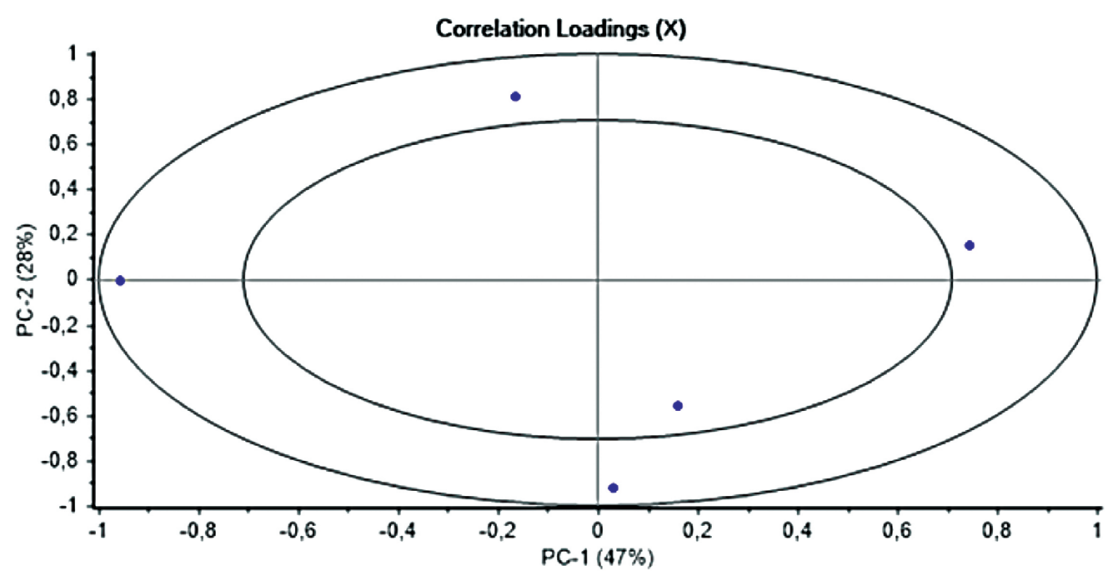

Figura 5. Gráfico de los pesos correlacionados resultado del ACP al fichero con 10 variables y 546 instancias

Además, el gráfico explica el fenómeno físico que se esperaba encontrar, ya que la variable temperatura, ubicada a la izquierda a lo largo del componente principal 1 (PC1) se encuentra relacionada de manera inversa con la humedad; lo cual es lógico ya que este componente es el que mayor influencia tiene en la variable de salida que es el \% de mermas en proceso. También se muestra la variable grado alcohólico que influye de manera negativa en el componente principal 2 (PC2), la cual tiene una relación inversa con la variable tiempo de añejamiento.

En el cuadro 5 se encuentran los pesos de cada una de las variables en los tres primeros componentes principales. Se subrayaron además las variables que mayor contribución tienen a cada componente principal; de esta manera el primer CP resultó estar relacionado a las variables medioambientales humedad y temperatura. El segundo CP se vincula al efecto del tiempo de añejamiento en el estudio y su repercusión en el grado alcohólico. Mientras que el tercer CP está mayormente influenciado por la humedad y el volumen, que son de cierta manera aleatorias en las muestras.

Cuadro 5. Pesos de las variables en cada componente principal

\begin{tabular}{|c|c|c|c|c|c|}
\hline Variables & CP1 & Variables & CP2 & Variables & CP3 \\
\hline Humedad $(\%)$ & $\underline{0,7440}$ & $\begin{array}{c}\text { Tiempo de } \\
\text { añejamiento (años) }\end{array}$ & $\underline{0,8125}$ & Volumen $(\mathrm{L})$ & $\underline{0,3778}$ \\
\hline Volumen $(\mathrm{L})$ & 0,1616 & Humedad $(\%)$ & $-0,1558$ & $\begin{array}{c}\text { Tiempo de añejamiento } \\
\text { (años) }\end{array}$ & $-0,1620$ \\
\hline Grado $\left({ }^{\circ} \mathrm{GL}\right)$ & 0,0326 & Temperatura $\left({ }^{\circ} \mathrm{C}\right)$ & $-0,0068$ & Temperatura $\left({ }^{\circ} \mathrm{C}\right)$ & $-0,2670$ \\
\hline $\begin{array}{c}\text { Tiempo de añejamiento } \\
(\text { años) }\end{array}$ & $-0,1638$ & Volumen $(\mathrm{L})$ & $-0,5577$ & Grado $\left({ }^{\circ} \mathrm{GL}\right)$ & $-0,2869$ \\
\hline Temperatura $\left({ }^{\circ} \mathrm{C}\right)$ & $-0,9551$ & Grado $\left({ }^{\circ} \mathrm{GL}\right)$ & $-\underline{0,9194}$ & Humedad $(\%)$ & $-0,6167$ \\
\hline
\end{tabular}

\section{Análisis económico}

Para la evaluación económica de los beneficios de este trabajo se calculan los porcentajes de pérdidas por mermas y se comparan con el 6 \% admisible en la Norma General No. 03-2012 de República Dominicana [9] ya que no existe una norma cubana aprobada. 
Los porcentajes de mermas se determinan teniendo en cuenta la diferencia entre los volúmenes final e inicial de los 45 toneles que se estudian. El precio de venta de ron es de $30 \$ /$ botella y cada botella contiene $700 \mathrm{~mL}$, quedando determinadas las pérdidas monetarias por concepto de merma en bodegas.

Las mermas en bodega durante los 13 meses de estudio fueron 763,43 L, lo cual equivale a un $10,5 \%$ del volumen inicial existente, que en comparación con lo establecido en la norma dominicana [9], para períodos de envejecimiento entre dos y cinco años (lo cual se ajusta a la mayoría de los datos existentes), supera el $6 \%$ permitido. Además, representa una pérdida de $\$ 32718$.

El valor de mermas admisible correspondiente al $6 \%$ establecido en la norma dominicana para la bodega, en este período de tiempo, es 434,72 L, que representa unas pérdidas monetarias de $\$ 18631$ y difiere del valor de pérdidas reales $\$ 14087$.

\section{Conclusiones}

Se determinaron cinco parámetros críticos que influyen en la calidad del proceso de añejamiento mediante un modelo de riesgo basado en matriz de entradas y salidas, estos fueron: humedad, temperatura, tiempo de añejamiento, concentración de dioxígeno disuelto y volumen medido.

Se realizó un análisis exploratorio de los datos que se miden en CERALBE, permitiendo reducir la dimensionalidad de los datos a tres componentes principales que explican el 89,11\% de la varianza, seleccionando las variables temperatura, humedad, tiempo de añejamiento, grado alcohólico y volumen para la modelación posterior.

Se calcularon las mermas en bodega durante los 13 meses de estudio, que resultaron ser 763,43 L, lo cual equivale a un 10,5\% del volumen inicial existente; superando el $6 \%$ permitido en la norma de República Dominicana y que representa una pérdida de \$32 718. Se determinó que el $6 \%$ de mermas con respecto al volumen inicial de la solera es 434,72 L, que representa unas pérdidas de \$18 631.

\section{Referencias}

[1] J. Pérez and R. Delgado, Bebidas alcohólicas destiladas y añejadas. [CD-ROM]. La Habana: IIIA, 2007.

[2] M. Boscolo, B. S. Lima-Neto, and D. W. Franco, "Envelhecimento de aguardente de cana-de-açucar em tonéis de madera", Engarrafador Moderno, vol. 6, no. 41, pp. 30-33, 1995.

[3] J. Marcano, (2019, Septiembre 15). El ro n. Tradición en Las Antillas [Online]. Available: https://mipais.jmarcano.com/economia/ron.html

[4] L. E. López, L. Zumalacárregui, O. Pérez, "Análisis de componentes principales aplicado a la fermentación alcohólica", Revista Científica de la UCSA, vol. 6, no. 2, pp. 11-19, 2019.

[5] Desiree, G. (2019, Septiembre 8). Conoce las etapas de validación de procesos [Online]. Available: https:// www.qbd.lat/blog/conoce-las-etapas-de-validacion-de-procesos

[6] K. Canter, "Utilizing RM in a submission for developing critical process parameters and critical to quality attributes", presented at FDA/Industry Statistics Workshop, Washington, D. C, 2006.

[7] M. D. González, M. A. Vázquez, D. Redondo, "Estudio comparativo de rones y aguardientes añejados en barriles de roble y por el proceso acelerado", ICIDCA, vol. XL, no. 3, pp. 12-19, 2006.

[8] L. Mesa, O. Gozá, M. Uranga, A. Toledo, and Y. Gálvez, "Aplicación del análisis de componentes principales en el proceso de fermentación de un anticuerpo monoclonal”, Vaccimonitor, vol. 27, no. 1, pp. 8-15, 2018.

[9] DGII (Dirección General de Impuestos Internos). (2019, Febrero 20). Norma general sobre el manejo del alcohol en las licorerías. No. 03-2012 [Online]. Available: https://www.dgii.gov.do/legislacion/normas/ Documents/03-2012.pdf 